Research Paper

\title{
A phase II trial of preoperative concurrent chemotherapy and dose escalated intensity modulated radiotherapy (IMRT) for locally advanced rectal cancer
}

\author{
Jeremy Tey ${ }^{\bowtie}$, Cheng Nang Leong1, Wai Kit Cheong33, Tay Guan Sze, Wei Peng Yong², Ivan Weng Keong \\ Tham ${ }^{1}$, Khai Mun Lee ${ }^{5}$ \\ 1. Department of Radiation Oncology, National University Cancer Institute, Singapore; \\ 2. Department of Medical Oncology, National University Cancer Institute, Singapore; \\ 3. Department of Colorectal Surgery, National University Hospital, Singapore; \\ 4. Department of Colorectal Surgery, Tan Tock Seng Hospital, Singapore; \\ 5. Department of Radiation Oncology, Farrer Park Hospital, Singapore. \\ $\square$ Corresponding author: Dr Jeremy Tey MD, Radiation Oncologist, Department of Radiation Oncology. National University Hospital, 5 Lower Kent Ridge \\ Road, Singapore 119074. Tel: +65 67724869; Fax: +65 67796320; Email: Jeremy_tey@nuhs.edu.sg \\ (c) Ivyspring International Publisher. This is an open access article distributed under the terms of the Creative Commons Attribution (CC BY-NC) license \\ (https://creativecommons.org/licenses/by-nc/4.0/). See http://ivyspring.com/terms for full terms and conditions.
}

Received: 2017.05.29; Accepted: 2017.08.11; Published: 2017.09.06

\begin{abstract}
Objectives: To determine the pathological response rates and toxicity and in patients with locally advanced rectal cancer treated with concurrent capecitabine and dose escalated intensity modulated radiotherapy (IMRT)

Methods: Patients with stage II or III adenocarcinoma of the rectum were treated with preoperative concurrent capecitabine and IMRT. Dose of capecitabine was $825 \mathrm{mg} / \mathrm{m}^{2}, 5$ days a week for 5 weeks. IMRT was used to deliver a dose of 45Gy in 25 fractions (1.8Gy per fraction daily, 5 days a week over 5 weeks) to the regional lymphatics and areas at risk of harbouring microscopic disease. A concomitant synchronous integrated boost (SIB) to the gross tumour with a margin to a total dose of 55Gy in 25 fractions was also delivered in the same period. TME surgery was performed 8 weeks after preoperative therapy. The primary endpoint is pathological complete response rate $(\mathrm{PCR})$ and the secondary endpoint was downstaging rates, Sphincter preservation rates (SPR), disease free survival (DFS) at 2 years and toxicity graded using the CTCAE v3.0.

Results: Twenty three patients were enrolled. Three were not evaluable; one did not complete treatment due to logistic issues and two declined surgery. The remaining 20 patients completed preoperative chemolMRT followed by TME surgery.

At a median follow-up of 38.2 months (17.5-53.2 months), $90 \%$ (18 of 20) patients were alive. The 2 year overall survival and DFS were $90 \%$ and $90 \%$ respectively. $35 \%(7 / 20)$ of patients had a PCR. $65 \%$ (13 of 20) patients had successful downstaging of their rectal tumours. There was no local recurrence. Sphincter preservation rate was $85 \%$. Treatment was well tolerated with only one patient (5\%) having Grade 3 radiation proctitis.

Conclusions: Preoperative concurrent capecitabine and dose escalated IMRT is well tolerated and results in high rates of $P C R$. A randomized trial comparing this regimen with standard 3D conformal chemoradiotherapy is warranted.
\end{abstract}

Key words: rectal Cancer, IMRT, preoperative, chemoradiotherapy, capecitabine

\section{Introduction}

Preoperative chemoradiotherapy is currently considered standard therapy for patients with locally advanced rectal cancer. The landmark German rectal cancer trial showed that preoperative 
chemoradiotherapy reduces local recurrence and acute/late radiation toxicity compared to postoperative chemoradiotherapy. ${ }^{1}$ The standard preoperative radiotherapy regimen is currently pelvic radiotherapy to a dose of 45-50.4 Gy in 25-28 fractions with concurrent 5 fluorouracil (5FU) based chemotherapy agent. Capecitabine, an oral prodrug of $5 \mathrm{FU}$ has been shown to be equivalent to $5 \mathrm{FU}$ in randomized studies and has increasingly been used with radiotherapy due to patient convenience. ${ }^{2,3}$ Studies have shown that a complete pathological response ( $\mathrm{pCR}$ ) after preoperative treatment have been associated with an improved outcome, both local control and overall survival. ${ }^{4,5}$ Strategies that have been employed to improve $\mathrm{pCR}$ rates include radiotherapy concurrent with combination chemotherapy such as 5FU/oxaliplatin ${ }^{6,7}$, or concurrent with combination chemotherapy combined with biologic agents such as vascular endothelial growth factor (VEGF) inhibitors such as bevacizumab $^{8}$. Neoadjuvant chemotherapy before chemoradiotherapy has also been studied as one of the strategies to improve $\mathrm{pCR}$ rates. ${ }^{9}$

Another strategy to increase $\mathrm{pCR}$ rates is radiotherapy dose escalation. Radiotherapy dose escalation studies have been shown that increasing radiotherapy doses in the preoperative treatment of rectal cancer lead to increased pCR rates. ${ }^{10}$ A recently published comparative effectiveness analysis showed that patients treated with higher radiotherapy doses are more likely to have negative nodes at surgery and be downstaged from cT3-T4 and/or node positive disease to ypT0-T2N0 after neoadjuvant chemoradiotherapy. ${ }^{11}$

The concern for radiotherapy dose escalation for rectal cancer is that the dose to the surrounding critical organs will be correspondingly increased, leading to increase to acute and late toxicities. The ability to spare organ at risks such as femoral heads, bladder and in particular small bowel is an important consideration if dose escalation to the primary tumour is to be attempted. Intensity modulated radiotherapy (IMRT) can potentially allow us to escalate the radiation dose to the primary rectal tumour whilst minimizing acute and late complications to organs at risk. Dosimetric studies have shown that IMRT leads to improved small bowel sparing when compared to 3-dimensional conformal radiotherapy (3DCRT). ${ }^{12}$ However, clinical data is lacking and there is a clear need for prospective studies to assess the clinical outcomes of patients treated with dose escalated IMRT.

The aim of this study is to determine the pCR rates and toxicities in patients with locally advanced rectal cancer treated with preoperative concurrent capecitabine and dose escalated intensity modulated radiotherapy (IMRT)

\section{Materials and Methods}

\section{Patients}

This review was approved by the institution review board, NHG DSRB Domain B.

Eligible patients had to meet the following criteria: Pathologically proven diagnosis of adenocarcinoma of the rectum, clinically staged as T3 or T4,N0-N2, and M0 -staged by MRI or transrectal ultrasound of the rectum. Patients were medically operable for resectable adenocarcinoma of the rectum at least $<12 \mathrm{~cm}$ from the anal verge with adequate liver/renal and haematological function, Eastern Cooperative Oncology Group (ECOG) performance $0-2$ and $\geq 18$ years of age. Exclusion criteria included prior invasive malignancy (except non-melanomatous skin cancer) unless disease free for a minimum of 3 years, prior systemic chemotherapy for colorectal cancer prior radiotherapy to the region of the study cancer that would result in overlap of radiation therapy fields, pregnancy or women of childbearing potential, extension of malignant disease into the anal canal.

\section{Endpoints}

The primary endpoint was pCR rates. Secondary endpoints included toxicity assessment, Downstaging rate: defined as a pathologic $\mathrm{T}$ and/or $\mathrm{N}$ stage that was lower than the clinical $\mathrm{T}$ and/or $\mathrm{N}$ stage, Sphincter preservation rate (SPR): defined as any procedure whereby the rectal tumor was removed while leaving behind the anal sphincter. A temporary colostomy was considered a sphincter-sparing procedure as long as the anal sphincter was spared. DFS at 2 years: defined as being alive with no evidence of rectal cancer recurrence, calculated from the end of chemoradiotherapy to date of event or last follow-up

\section{Radiotherapy}

\section{Localization, Simulation, and Immobilization}

A treatment planning CT scan will be performed to define the gross tumor volume (GTV), clinical target volume (CTV), and planning target volume (PTV). Contiguous CT slices of $3 \mathrm{~mm}$ thickness of the abdomen would be acquired with the patient in the prone position in a belly board. No contrast is required. Patients should be simulated with a full bladder.

The elective CTVs are generated in accordance to the RTOG anorectal contouring guidelines (https://www.rtog.org/CoreLab/ContouringAtlases 
/Anorectal.aspx). The GTV-P (Primary) is the rectal tumour seen on CT/MRI scans. The GTV-N is the grossly enlarged lymph nodes seen on CT/MRI. The CTV-P (primary) is the GTV-P plus $2 \mathrm{~cm}$ margin, ensuring to extend to the entire mesorectum and presacral region at involved levels. The CTV-N is the GTV-N plus a $5 \mathrm{~mm}$ margin. Presacral lymphatic CTV is generated by contouring from mid S1-S5 and $8 \mathrm{~mm}$ tissue anterior to the anterior border of the sacral bone. The mesorectum and perirectal lymphatics CTV is generated by utilizing the following anatomic landmarks: Posterior Border: anterior border of the sacrum and gluteus maximus, Lateral Border: ileum, piriformis and obturator muscles, Anterior Border: should overlap by $1 \mathrm{~cm}$ into the bladder, vagina or prostate. The PTV45 is generated by expanding all of the above structures by $0.5 \mathrm{~cm}$ symmetrically and unifying them into one 3-dimensional volume for planning purposes. PTV 50 is the CTV-P plus a $5 \mathrm{~mm}$ margin. PTV 55 includes GTV-P plus $1 \mathrm{~cm}$ margin and CTV-N plus $5 \mathrm{~mm}$ margin. Megavoltage cone beam CT scans are performed daily prior to treatment for treatment verification. Figure 1 . shows the ability of
IMRT to conform radiation doses and 'dose paint' to target volumes.

\section{Treatment Planning and Dose specifications}

Treatment plans for patients will consist of a single phase using a synchronous integrated boost technique (SIB): Inverse-planned IMRT- will deliver to PTV 45 a total dose of 45 Gy in 1.8 Gy daily fractions, to PTV 50 a total dose of 50 Gy in 2 Gy daily fractions and to PTV 55 a total dose of 55 Gy in 2.2 Gy daily fractions. Patients will receive treatment 5 days per week, in once daily fractions, over 5 weeks duration. Dose is prescribed according to ICRU 83. Dose constraints to organs at risk are as follows: Small bowel - no more than $180 \mathrm{cc}$ above $35 \mathrm{~Gy}$, no more than $100 \mathrm{cc}$ above $40 \mathrm{~Gy}$, no more than $65 \mathrm{cc}$ above 45 Gy, no small bowel volume should receive $50 \mathrm{~Gy}$. Femoral heads- no more than $40 \%$ volume above $40 \mathrm{~Gy}$. no more than $25 \%$ above $45 \mathrm{~Gy}$, no femoral head volume should receive 50Gy. Bladder- no more than $40 \%$ volume above $40 \mathrm{~Gy}$, no more than $15 \%$ above $45 \mathrm{~Gy}$, no bladder volume should receive 50 Gy.

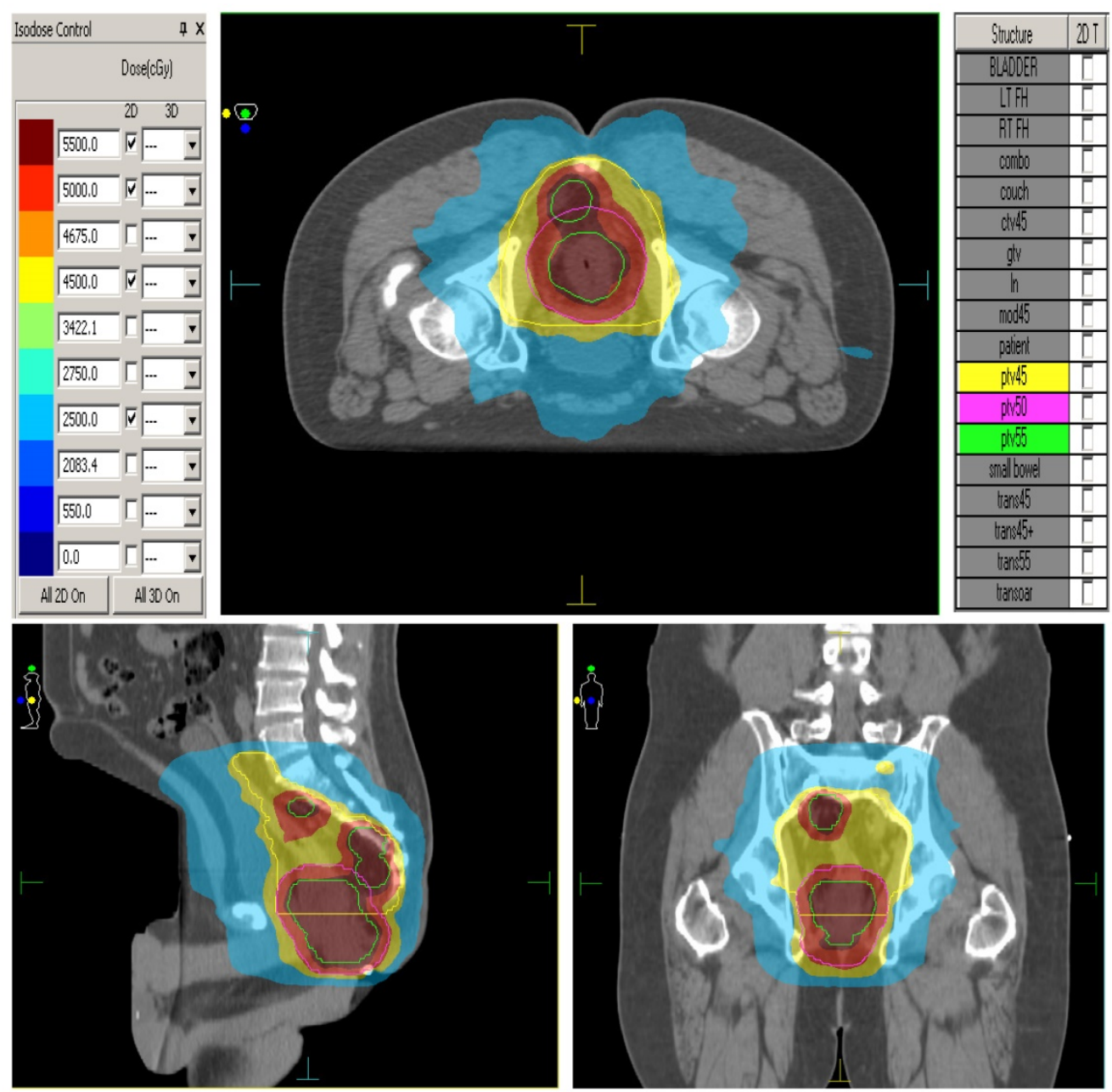

Figure 1. Dose painting with IMRT 


\section{Chemotherapy}

Concurrent preoperative chemotherapy will begin on the first day of radiotherapy and continue until the completion of radiation therapy. Capecitabine $\left(825 \mathrm{mg} / \mathrm{m}^{2}\right)$ will be administered orally twice daily 5 days a week during radiotherapy. Dose calculations should be based upon actual body weight and not modified for obesity. Postoperative chemotherapy will be administered at the discretion of the treating medical oncologist.

\section{Surgery}

Total mesorectal excision (TME) surgery was performed at least 8 weeks after preoperative therapy. Surgery includes abdominal perineum resection (APR) or an anterior resection (AR). The nature of the planned surgery must be documented the time of study entry.

\section{Pathological assessment}

Tumour response post-surgery will be grading according to the tumour regression grading ${ }^{13}$. TRG 0 is defined as complete pathological response. TRG 1 is marked response (minimal response with singe or small groups of cancer cells). TRG 2 is moderate response (residual cancer outgrown by fibrosis). TRG 3 is poor or no response (extensive residual cancer). A positive surgical margin is defined as tumour present $1 \mathrm{~mm}$ or less from the resection margin

\section{Safety parameters}

This study will use the International Common Terminology Criteria for Adverse Events (CTCAE) version 3.0 for toxicity and adverse event reporting. Toxicities were evaluated and recorded weekly. Safety and toxicity of the study treatment will be evaluated by clinical examination, haematological evaluation as well as imaging studies (MRI or CT) where indicated.

\section{Patient follow-up}

Patient would be followed up one month after completion of chemoradiotherapy, and thereafter every 3 monthly. History and physical examination would be performed at each follow-up, together with adverse event evaluation. CT chest/abdomen/pelvis would be performed annually for five years.

\section{Statistical analysis and trial registration}

This is a phase 2 single arm study. Sample size calculation is based on the following parameters: The Null hypothesis is that the IMRT dose escalation is no better than 3DCRT, with a pCR rate of $8 \%$. The alternate hypothesis is that IMRT dose escalation is better than 3DCRT, with a pCR rate of $25 \%$. Type I error rate is 0.05 Type II error rate is 0.20 . Using the "Minimax" two-stage design suggested by Simon ${ }^{14}$, 12 patients will be accrued in the first stage. If no $\mathrm{pCR}$ occurs in these 12 patients, the study will be terminated because of lack of efficacy. Otherwise, another 36 patients would be accrued to complete the study of 48 patients. Allowing for a $10 \%$ drop out rate, the target accrual will be 53 patients.

\section{Results}

Between January 2011 and August 2013, 23 patients were enrolled. Patient and tumour characteristics are detailed in table 1 . Three patients were not evaluable. One patient had 2 fractions of treatment and did not complete the remaining 23 fractions due to logistical issues. Two patients declined surgery after completion of chemoradiotherapy, citing that their rectal symptoms have resolved post treatment and they did not see a need for surgery. For these two patients, MRI post chemoradiotherapy revealed a partial clinical response. These two patients defaulted subsequent follow-up. The remaining 20 patients completed preoperative chemo-IMRT followed by TME surgery.

Table 1. Patient and tumour characteristics?

\begin{tabular}{lll}
\hline Patient Characteristics & $\mathrm{N}$ & $\%$ \\
\hline Gender & 14 & 70 \\
Male & 6 & 30 \\
Female & & \\
Age & 2 & 10 \\
$\leq 50$ & 18 & 90 \\
$>50$ & & \\
Location of tumour & 0 & 0 \\
Upper(8-12cm) & 8 & 40 \\
Mid $(4-8 \mathrm{~cm})$ & 12 & 60 \\
Lower $(0-4 \mathrm{~cm})$ & & \\
cT & 1 & 5 \\
T2 & 18 & 90 \\
T3 & 1 & 5 \\
T4 & & \\
cN & 2 & 10 \\
N0 & 17 & 85 \\
N1 & 1 & 5 \\
N2 & & \\
Combine clinical stage & 1 & 5 \\
T2N1 & 2 & 10 \\
T3N0 & 15 & 75 \\
T3N1 & 1 & 5 \\
T3N2 & 1 & 5 \\
T4N1 & & \\
\hline
\end{tabular}

Ninety percent of patients were over the 50 years of age (median age 59.5 years. Range 48-74 years). Location of all tumours was either in the mid or lower rectum. Majority of patients had cT3 and cN1 disease. The mean distance from the anal verge was $5.4 \mathrm{~cm}$ (range 2-8 cm). Four of 12 patients achieved a pCR 
fulfilling the requirements of the first stage of accrual, allowing the study to proceed to the second stage of accrual. 3 of 8 patients achieved a pCR in the second stage of accrual. The study had to be closed due to slow accrual and withdrawal of funding support. At a median follow-up of 38.2 months (17.5-53.2 months), $90 \%$ (18 of 20) patients were alive. The 2 year DFS was $90 \%$. There was no local recurrence.

\section{Treatment compliance}

Nineteen patients (95\%) completed radiotherapy with no treatment break. One patient had a 2 day break because of symptomatic prolapsed haemorrhoids and G3 radiation proctitis. All patients completed planned treatment. There were no patients who required replanning of their radiation treatment. No patients had a break in the chemotherapy during concurrent chemoradiotherapy

\section{Radiotherapy Quality assurance}

Individual treatment parameters such as fractionation, total dose, CTV contouring and OAR constraints were reviewed relative to protocol specifications. Compliance to protocol was excellent with no treatment deviation.

\section{Toxicity}

Treatment was well tolerated. One patient $(5 \%)$ had grade 3 acute radiation proctitis, and one patient (5\%) had grade 3 late radiation proctitis. This patient had radiation proctitis and presented with per rectal bleeding. She was treated with argon plasma coagulation. Her proctitis resolved one year after treatment and she is still on follow-up. Acute toxicities for all patients are shown in Table 3. There were no hematological side effects.

\section{Surgery}

The median time to surgery was 60 days (range 49-85 days). Eighty percent of patients underwent surgery by 9 weeks after chemoradiotherapy. $20 \%$ of patients $(4 / 20)$ underwent surgery after 9 weeks. Of these 4 patients, only one patient had a pCR. There was no surgical morbidity, in particular anastomotic leak or wound infection. The median hospital stay was 6 days (range 3-12 days). Surgical procedures performed and pathological findings are detailed in table 2. Low anterior resections were performed in 17 patients and abdominal perineal resection in 3 patients. Sphincter preservation rate was $85 \%$. Preoperative chemoradiotherapy did not change the type of surgery documented at study entry.

\section{Pathological response}

Thirty five percent $(7 / 20)$ of patients had a pCR (TRG 1). 65\% (13 of 20) patients had successful downstaging of their rectal tumours (6 patients had TRG 1 and 7 patients had TRG 2). Nineteen patients had clear surgical margins. One patient $(5 \%)$ had a positive margin post-surgery.

\section{Post-operative chemotherapy}

Nineteen of 20 patients received postoperative chemotherapy. 12 patients had adjuvant capecitabine only, whilst 7 patients had adjuvant capecitabine with oxaliplatin chemotherapy.

Table 2. Surgical procedure and pathological Findings

\begin{tabular}{lll}
\hline & $\mathrm{N}$ & $\%$ \\
\hline Surgery & 17 & \\
Low Anterior resection & 3 & 15 \\
Abdominal Perineal resection & & \\
Lymphovascular invasion (LVI) & 1 & 5 \\
Yes & 19 & 95 \\
No & & \\
Perineural invasion & 2 & 10 \\
Yes & 18 & 90 \\
No & & \\
Margin & 1 & 5 \\
Negative & 19 & 95 \\
Positive & & \\
ypT & 7 & 35 \\
T0 & 0 & 0 \\
T1 & 5 & 25 \\
T2 & 8 & 40 \\
T3 & 0 & 0 \\
T4 & & \\
ypN & 14 & 70 \\
N0 & 5 & 25 \\
N1 & 1 & 5 \\
N2 & & \\
Tumour regression grading (TRG) & 7 & 35 \\
0 & 6 & 30 \\
1 & 7 & 35 \\
2 & 0 & \\
3 & & \\
\hline
\end{tabular}

\section{Discussion}

The results of our study show that preoperative concurrent capecitabine and dose escalated radiotherapy delivered using IMRT resulted in a high pCR of $35 \%$ compared to up to $25 \%$ in studies using standard dose 3 dimensional conformal radiotherapy (3DCRT). 1,15,16 We treated the primary tumour to a dose of 55Gy in 25 fractions, whilst treating the pelvic PTV to the conventional dose of 45Gy in 25 fractions. Using a SIB technique, we were able to increase the radiation dose to the primary tumour whilst shortening the treatment time from the conventional 28 days to 25 days. Such dose escalation may lead to increased dose to OARs and therefore increased toxicity to the small bowel, bladder and femoral heads. The toxicity rates with our treatment however, were low, with one patient $(5 \%)$ having grade 3 radiation proctitis post-surgery. The low rates of $\geq$ 
grade 3 toxicity are likely due to the use of IMRT in our study. IMRT is already the standard of care in the treatment of many cancers, including prostate and nasopharyngeal cancers. ${ }^{17,18}$ In one of the earliest dosimetric studies, (2006) Urbano et al reported a comparison of 3DCRT with IMRT in patients with locally advanced rectal cancer. ${ }^{12}$ He showed that the bowel volume irradiated to 45Gy and 50Gy was significantly reduced with IMRT. In our study, despite increasing the dose to the primary tumour, we were still able to spare the OARs. It is our opinion that the value of IMRT for rectal cancer lies in the ability to allow dose escalation to the primary tumour whilst sparing the OARs.

Table 3. Acute toxicity for all patients

\begin{tabular}{|c|c|c|}
\hline Toxicity (CTC v3.0) & Number & $\%$ \\
\hline \multicolumn{3}{|l|}{ Nausea } \\
\hline G0 & 16 & 80 \\
\hline G1 & 3 & 15 \\
\hline G2 & 1 & 5 \\
\hline \multicolumn{3}{|l|}{ Vomiting } \\
\hline G0 & 19 & 95 \\
\hline G1 & 0 & 0 \\
\hline G2 & 1 & 5 \\
\hline \multicolumn{3}{|l|}{ Anorexia } \\
\hline G0 & 13 & 65 \\
\hline G1 & 7 & 35 \\
\hline G2 & 0 & 0 \\
\hline \multicolumn{3}{|l|}{ Proctitis } \\
\hline G0 & 14 & 70 \\
\hline G1 & 4 & 20 \\
\hline G2 & 1 & 5 \\
\hline G3 & 1 & 5 \\
\hline \multicolumn{3}{|l|}{ Dermatitis } \\
\hline G1 & 9 & 45 \\
\hline G1 & 6 & 30 \\
\hline G2 & 5 & 25 \\
\hline \multicolumn{3}{|l|}{ Diarrhoea } \\
\hline G0 & 11 & 55 \\
\hline G1 & 7 & 35 \\
\hline G2 & 2 & 10 \\
\hline \multicolumn{3}{|l|}{ Urinar } \\
\hline G0 & 12 & 60 \\
\hline G1 & 5 & 25 \\
\hline G2 & 3 & 15 \\
\hline \multicolumn{3}{|l|}{ Haematological } \\
\hline G0 & 20 & 100 \\
\hline G1 & 0 & 0 \\
\hline G2 & 0 & 0 \\
\hline
\end{tabular}

Other prospective trials have been conducted to investigate the use of IMRT in the preoperative treatment of rectal cancer. In an attempt to reduce GI toxicity, RTOG 0822 was designed to investigate the rate of GI toxicity with preoperative IMRT delivered with capecitabine and oxaliplatin for patients with locally advanced rectal cancer. ${ }^{19}$ Hong et al reported that the use of IMRT in this prospective phase 2 trial study did not reduce the rate of GI toxicity.
Unfortunately, interpretation of this study is limited by the use of concurrent oxaliplatin with 5-FU based chemoradiation, as there are four large, phase III randomized trials that have demonstrated increased GI toxicity when oxaliplatin is added to pre-operative chemoradiation. ${ }^{20-23}$

The dose fractionation regimen of 55 Gy in 25 fractions represents an approximate $10 \%$ increase in radiation dose compared with the conventionally used regimen of $50.4 \mathrm{~Gy}$ in 28 fractions. This increase in radiation dose is likely to have led to increased $\mathrm{pCR}$ rates in our study compared to studies of conventional dose fractionation regimens reported in the literature. Indeed, (2006) Wiltshire et al investigated in 3 prospective phase II trials the effect of radiotherapy dose escalation on pCR rates. ${ }^{10}$ It was found that there was a trend of increasing $\mathrm{PCR}$ rates ranging from 15 to $33 \%$ with increasing RT doses of 40 Gy, 46 Gy, and 50 Gy in 2 Gy fractions. The increase in pCR rates was associated with a significant increase in local relapse free survival, DFS and OS.

Two other studies reported IMRT dose escalation using similar dose fractionation regimens. The University Colorado Cancer Centre (UCCC) ${ }^{24}$ and the Fox Chase Cancer Centre (FCCC) ${ }^{25}$ studies treated patient with operable stage II and III adenocarcinoma of the rectum with preoperative IMRT to a total dose of 55Gy in 25 fractions, concurrent with capecitabine $825 \mathrm{mg} / \mathrm{m}^{2}$. Accrual was poor in both studies with only 8 patients in each study. The UCCC study reported a crude pCR rate of $38 \%$ and grade 4 diarrhoea in 1 of $8(13 \%)$ patients. In contrast, the FCCC study reported unacceptable toxicities rates in 3 of $8(38 \%)$ of patients, with no patient having pCR.

The major criticisms of the FCCC study are that patients were treated with capecitabine for 7 days each week during radiation, which might have contributed to increase rates of grade 3 gastrointestinal toxicity. In addition, the pelvic CTV was expanded by $1 \mathrm{~cm}$ to obtain the pelvic PTV, and the GTV by $2 \mathrm{~cm}$ to obtain the primary tumour PTV. These large PTV expansions might have contributed to increased volumes of bowel irradiated. In addition, OAR constraints were not provided by the FCCC study.

We report outcomes similar to the results of the UCCC study. In our study, we treated patients to 3 radiation dose levels with different PTV expansion margins. PTV 45 was the pelvic CTV with a $5 \mathrm{~mm}$ expansion. PTV 50 was obtained with the conventional $2.5 \mathrm{~cm}$ expansion from GTV. PTV 50 was generated in addition to PTV 55/PTV 45 used in the FCCC/UCCC studies because our team felt that this minimized the risk of geographical miss in the boost volume and ensured that the primary rectal 
tumour received at least 50Gy in 25 fractions. PTV 55 was obtained with a $1 \mathrm{~cm}$ expansion from the GTV. The smaller margins used may have accounted for less bowel irradiated and hence lower GI toxicities. IMRT allowed us to use a SIB technique to deliver different daily dose per fraction to all the PTVs. The use of daily IGRT for all our patients allowed us to reduce the PTV expansions to $5 \mathrm{~mm}$. In addition, we treated all patients in the prone position with a belly board. Dosimetric studies have shown that the use of a belly board with IMRT provides better small bowel sparing when compared with a belly board with 3DCRT. ${ }^{26,27}$ Patients treated also received capecitabine 5 days a week during radiotherapy instead of 7 days a week. These factors may also have led to reduced toxicities in our study.

Finally, there is emerging data that patients can avoid surgery if they can achieve a clinical complete response (cCR) to chemoradiotherapy. Data from Brazilian and Dutch studies have shown excellent outcomes in patients who achieved CCR after chemoradiotherapy and followed up closely after. ${ }^{28,29}$ With increased pCR rates with IMRT dose escalation, select patients can potentially have avoidance of surgery. The challenge is accurate non operative identification of patients who are complete or near complete responders. This organ preservation approach is promising and is currently being validated in prospective clinical trials.

The strengths of our study are: Firstly this study standardized the contouring of clinical target volumes according to the RTOG anorectal contouring guidelines. This ensures consistency and reproducibility of target volumes which allows meaning comparisons of results with other studies. Secondly, our study affirms the results of the UCCC trial. Given the high pCR rates and favorable toxicity of IMRT dose escalation, we are currently planning a phase 2 randomized trial comparing IMRT dose escalation with convention 3DCRT. Thirdly, radiotherapy quality assurance was performed for every patient within one week of patient starting treatment. The limitation of our study is that we did not manage to accrue the planned total number of patients due to the withdrawal of funding support. In light of the FCCC study, although patients with high rectal tumours were eligible for study entry, we recruited patients with exclusively mid and lower rectal cancer. This limited the number of patients accrued in our study and also highlights the consideration of our group that high rectal tumours may not be suitable for radiotherapy dose escalation compared to mid/lower tumours because of their proximity to colon and small bowel.

\section{Conclusion}

Our study shows that preoperative concurrent capecitabine and dose escalated IMRT is well tolerated and results in high $\mathrm{pCR}$ rates. A randomised trial comparing this regimen with standard $3 \mathrm{D}$ conformal chemoradiotherapy is warranted.

\section{Acknowledgements}

This research is supported by the Singapore Ministry of Health's National Medical Research Council under its New Investigator Grant (NMRC NIG09nov046.).

\section{Competing Interests}

The authors have declared that no competing interest exists.

\section{References}

1. Sauer R, Becker H, Hohenberger W, et al. Preoperative versus postoperative chemoradiotherapy for rectal cancer. N Engl J Med. 2004; 351:1731-40.

2. Twelves CS, Gollins S, Grieve R, et al. A randomized cross-over trial comparing patient preference for oral capecitabine and 5-fluorouracil/leucovorin regimens in patients with advanced colorectal cancer. Ann Oncol. 2006;17(2):239-45.

3. Twelves C, Wong A, Nowacki MP, et al. Capecitabine as adjuvant treatment for stage III colon cancer. N Engl J Med. 2005;352(26):2696-704.

4. Monique Maas, PJ Nelemans, V Valentini et al. Long term outcome in patients with a pathological complete response after chemoradiation for rectal cancer: A pooled analysis of individual patient data. Lancet Oncol. 2010; 11:835-44.

5. Carlo Capirci, Vincenzo Valentini, Luca Cionini et al. Prognostic value of complete pathologic complete response after neoadjuvant therapy in locally advanced rectal cancer: Long Term Analysis of 566 ypCR patients. Int J Rad Oncol Biol Physics. 2008; 72(1):99-107

6. Lu JY, Xiao Y, Qiu HZ. Clinical outcome of neoadjuvant chemoradiation therapy with oxaliplatin and capecitabine or 5-fluorouracil for locally advanced rectal cancer. J Surg Oncol. 2013;108(4):213-9.

7. Zhao L, Bai C, Shao Y, et al. A phase II study of neoadjuvant chemoradiotherapy with oxaliplatin and capecitabine for rectal cancer. Cancer Lett. 2011;310(2):134-9

8. Miki Y, Maeda K, Hosono M, et al. Neoadjuvant capecitabine, bevacizumab and radiotherapy for locally advanced rectal cancer: results of a single-institute Phase I study. J Radiat Res. 2014;55(6):1171-7

9. Cercek A, Goodman KA, Hajj C, et al. Neoadjuvant chemotherapy first, followed by chemoradiation and then surgery, in the management of locally advanced rectal cancer. J Natl Compr Canc Netw. 2014;12(4):513-9.

10. Kirsty L. Wiltshire, Iain G. Ward, Carol Swallow et al. Pre-operative radiation with concurrent chemotherapy for respectable rectal cancer: Effect of dose escalation on pathologic complete response, local recurrence- free survival, disease-free survival, and overall survival. Int J Rad Oncol Biol Physics. 2006; 64(3):709-716.

11. Hall MD, Schultheiss TE, Smoth DD, et al. Effect of increasing radiation doe on pathologic complete response in rectal cancer patients treated with neoadjuvant chemoradiation therapy. Acta Oncol. 2016;55(12):1392-1399

12. Urbano MTG, Henrys AJ, Adams EJ, et al. Intensity-modulated radiotherapy in patients with locally advanced rectal cancer reduces volume of bowel treated to high dose levels. Int J Radiat Oncol Biol Phys. 2006;65:907-9

13. Ryan $R$, et al. Pathological response following long-course neoadjuvant chemoradiotherapy for locally advanced rectal cancer. Histopathology. 2005;47(2):141-146

14. Simon R. Optimal Two-Stage Designs for Phase II Clinical Trials. Controlled Clinical Trials. 1989; 10:1-10.

15. Gerard J, Conroy T, Bonnetain F, et al. Preoperative radiotherapy with or without fluorouracil and leucovorin in T3-4 rectal cancers: Results of FFCD 9203. J Clin Oncol. 2006; 24:4620-4625.

16. Bujko K, Nowacki MP, Nasierowska A, et al. Long-term results of a randomized trial comparing preoperative short-course radiotherapy with preoperative conventionally fractionated chemoradiation for rectal cancer. $\mathrm{Br} \mathrm{J}$ Surg. 2006; 93:1215-1223.

17. Schroeck FR, Jacobs BL, Bhayani SB, et al Cost of New Technologies in Prostate Cancer Treatment: Systematic Review of Costs and Cost Effectiveness of Robotic-assisted Laparoscopic Prostatectomy, Intensity-modulated Radiotherapy, and Proton Beam Therapy. Eur Urol. 2017;(S):0302-2838(17)30239-7 
18. Lu Z, Chen QY, Liu H, et al. Emerging treatment options for nasopharyngeal carcinoma. Drug Des Devel Ther. 2013; 7:37-52

19. Hong TS, Mouqhan J, Garofalo MC, et al. NRG Oncology Radiation Therapy Oncology Group 0822: A Phase 2 Study of Preoperative Chemoradiation Therapy Using Intensity Modulated Radiation Therapy in Combination With Capecitabine and Oxaliplatin for Patients With Locally Advanced Rectal Cancer. Int J Radiat Oncol Biol Phys. 2015; 93(1):29-36.

20. Aschele C, Cionini L, Lonardi S, et al. Primary tumor response to preoperative chemoradiation with or without oxaliplatin in locally advanced rectal cancer: pathologic results of the STAR-01 randomized phase III trial. J ClinOncol. $2011 ; 29: 2773-2780$

21. Gerard JP, Azria D, Gourgou-Bourgade S, et al. Comparison of two neoadjuvant chemoradiotherapy regimens for locally advanced rectal cancer: results of the phase III trial ACCORD 12/0405-Prodige 2. J ClinOncol. 2010; 28:1638-1644.

22. O'Connell MJ, Colangelo LH, Beart RW, et al. Capecitabine and oxaliplatin in the preoperative multimodality treatment of rectal cancer: surgical end points from National Surgical AdjuvantBreast and Bowel Project trial R-04. J ClinOncol. 2014;32(18):1927-1934.

23. Rodel C, Liersch T, Becker H, et al. Preoperative chemoradiotherapy and postoperative chemotherapy with fluorouracil and oxaliplatin versus fluorouracil alone in locally advanced rectal cancer: Initial results of the German CAO/ARO/AIO-04 randomized phase 3 trial. Lancet Oncol. 2012; 13:679-687.

24. Ari Ballonoff, Brian Kavanagh, Martin McCarter et al. Preoperative Capecitabine and Accelerated Intensity Modulated radiotherapy in locally advanced rectal cancer: A Phase II trial. Am J ClinOncol. 2008;31(3):264-270

25. Freedman GM, Meropol NJ, Sigurdson ER, et al. Phase I trial of preoperative hypofractionated intensity-modulated radiotherapy with incorporated boost and oral capecitabine in locally advanced rectal cancer. Int J Radiat Oncol Biol Phys. 2007;67: 1389 -1393.

26. Cranmer-Sargison G, Kundapur V, Park-Somers E et al. Planning target volume margin evaluation and critical structure sparing for rectal cancer patients treated prone on a belly board. Clin Oncol ( $\mathrm{R}$ Coll Radiol). 2013;25(3e):17-22

27. Nijkamp J, Doodeman B, Marijnen C, et al. Bowel exposure in rectal cancer IMRT using prone, supine, or a belly board. Radiother Oncol. 2012;102(1):22-9

28. Habr-Gama A, Perez RO, Nadalin $\mathrm{W}$, et al. Operative versus nonoperative treatment for stage 0 distal rectal cancer following chemoradiation therapy: long-term results. Ann Surg. 2004;240(4):711-7.

29. Maas M, Beets-Tan RG, Lambregts DM, et al. Wait-and-see policy for clinical complete responders after chemoradiation for rectal cancer. J Clin Oncol 2011; 29:4633-40 